\title{
False Replies on Smoking Rate Surveys and an In-Hospital Smoking Cessation Clinic
}

\author{
Jungun Lee \\ Department of Family Medicine, Wonkwang University Sanbon Hospital, Wonkwang University School of Medicine, Gunpo, Korea
}

Cigarette smoking is a leading risk factor for cancer and cardiovascular and pulmonary disorders. It has been recommended that primary care physicians inquire about a patient's smoking status and recommend smoking cessation during every medical consultation. ${ }^{1)}$ Thus, smoking rates and smoking cessation have been major topics in papers published in the Korean Journal of Family Medicine.

In this issue, Kim et al. ${ }^{2)}$ report a discrepancy between selfreporting and urine cotinine-verified smoking status in South Korea. Among 3,477 men, urine cotinine-verified smoking rates were $11.1 \%$ higher than the self-reported smoking rate. About 1 in 3 participants did not complete a smoking questionnaire or gave false replies. Age greater than 60 years, an educational level of high school graduation or lower, multiple health check-ups, and urine cotinine levels $<500 \mathrm{ng} / \mathrm{mL}$ were associated with a discrepancy between the smoking rates in the self-reported and cotinine-verified groups. This study was performed in a single hospital unit. However, the results were similar to those of previous studies, which showed that, compared to smoking rates determined by counter-verification with biomarkers, smoking rates in self-reports are lower in populations that are pressured to abstain from cigarette smoking for medical or social reasons, such as pregnant women, ${ }^{3,4)}$ teenagers, ${ }^{5)}$ those undergoing treatment for smoking cessation, ${ }^{6)}$ and those with pulmonary disorders. ${ }^{7)}$

Another study by Shin et al. ${ }^{\text {8) }}$ presented the results of compliance and effectiveness of a smoking cessation program started in hospitalized patients. Of these, $74.5 \%$ were cardiovascular disease patients who were referred from neurology and cardiology and who needed to stop smoking for secondary prevention. However, only $67.9 \%$ of the referred patients actually had a consultation and prescription. Moreover, even those patients who were prescribed a medicine to quit smok- ing through a consultation rarely revisited the outpatient clinic after discharge from the hospital. This study indicated that there were various obstacles to treatment in terms of referring hospitalized patients to the smoking cessation clinic. The authors state that it is imperative to introduce a systematic smoking cessation program, which emphasizes the relationship between smoking and disease, for hospitalized patients and to encourage cooperation to improve the smoking cessation rate.

After the implementation of a new tax policy that raised the cigarette price in South Korea, smoking cessation treatment gained support from the National Health Insurance Program. Under these circumstances, primary care physicians are likely to encounter more patients with tobacco addiction problems. Therefore, they should be familiar with smoking cessation counseling and treatment regimens.

\section{CONFLICT OF INTEREST}

No potential conflict of interest relevant to this article was reported.

\section{REFERENCES}

1. National Center for Chronic Disease Prevention and Health Promotion (US) Office on Smoking and Health. The health consequences of smoking: 50 years of progress: a report of the surgeon general. Atlanta (GA): Centers for Disease Control and Prevention (US); 2014.

2. Kim Y, Choi YJ, Oh SW, Joh HK, Kwon H, Um YJ, et al. Discrepancy between self-reported and urine-cotinine verified smoking status among Korean male adults: analysis of health check-up data from a single private hospital. Korean J Fam Med 2016;37:171-6. 
3. Lee DR, Kim HS, Lee J. The characteristics of false respondents on a self-reported smoking survey of Korean women: Korean National Health and Nutrition Examination Survey, 2008. Korean J Fam Med 2014;35:28-34.

4. Webb DA, Boyd NR, Messina D, Windsor RA. The discrepancy between self-reported smoking status and urine continine levels among women enrolled in prenatal care at four publicly funded clinical sites. J Public Health Manag Pract 2003;9:322-5.

5. Jeong IS, Park NR, Ham J. Agreement between smoking self-report and urine cotinine among adolescents. J Prev Med Public Health 2004;37: 127-32.
6. Kim JY, Kwon M, Joe KH, Chai SH, Won WY, Kim DJ. Agreement between self-report on smoking cessation and urinary cotinine test. Korean J Psychopharmacol 2010;21:210-5.

7. Murray RP, Connett JE, Lauger GG, Voelker HT. Error in smoking measures: effects of intervention on relations of cotinine and carbon monoxide to self-reported smoking. The Lung Health Study Research Group. Am J Public Health 1993;83:1251-7.

8. Shin GH, Yi SW, Cheong YS, Park EW, Choi EY. Compliance and effectiveness of smoking cessation program started on hospitalized patients. Korean J Fam Med 2016;37:149-55. 